\title{
Sleep-related breathing disorders and non-invasive ventilation
}

\author{
Agata Lax, ${ }^{1}$ Simona Colamartino, ${ }^{2}$ Paolo Banfi, ${ }^{1}$ Antonello Nicolini ${ }^{2}$ \\ ${ }^{1}$ Neuromuscular Diseases Unit, Don Gnocchi Foundation, Milan; ${ }^{2}$ Respiratory Medicine Unit, Hospital of Sestri Levante \\ (GE), Italy
}

\begin{abstract}
Non-invasive mechanical ventilation (NPPV) was originally used in patients with acute respiratory impairment or exacerbations of chronic respiratory diseases, as an alternative to the endotracheal tube. Over the last thirty years NPPV has been also used at night in patients with stable chronic lung disease such as obstructive sleep apnea, the overlap syndrome (chronic obstructive pulmonary disease and obstructive sleep apnea), neuromuscular disorders, obesity-hypoventilation syndrome, and in other conditions such as sleep disorders associated with congestive heart failure (Cheyne-Stokes respiration). In this no-systematic review we discuss the different types of NPPV, the specific conditions in which they can be used and the indications, recommendations and evidence supporting the efficacy of NPPV. Optimizing patient acceptance and adherence to non-invasive ventilation treatment is challenging. The treatment of sleep-related disorders is a life-threatening condition. The optimal level of treatment should be determined in a sleep laboratory. Side effects directly affecting the patient's adherence to treatment are known. The most common are nasopharyngeal symptoms including increased congestion and rhinorrhea; these effects are related to reduced humidity of inspired gas. Humidification of delivered gas may improve these symptoms.
\end{abstract}

\section{Introduction}

Non-invasive mechanical ventilation (NPPV) was originally used in patients with acute respiratory impairment or exacerbations of chronic respiratory diseases, as an alternative to the endotracheal tube. Over the last thirty years NPPV has been also used at night

Correspondence: Nicolini Antonello, Respiratory Diseases Unit, Hospital of Sestri Levante, via Terzi 43, 16039 Sestri Levante (GE), Italy.

Tel.: +39.0185.329145 - Fax: +39.0185.329935.

E-mail: antonello.nicolini@fastwebnet.it

Key words: Sleep-related respiratory disorders; non-invasive ventilation; continuous positive airway pressure; bilevel positive airway pressure.

Contributions: AL drafted and revised the manuscript; SC revised the manuscript; PB designed the review, drafted and revised the manuscript; AN designed the review, drafted and revised the manuscript.

Conflict of interest: the authors declare that they have no conflict of interest.

Received for publication: 1 June 2013.

Revision received: 24 October 2013.

Accepted for publication: 25 November 2013.

This work is licensed under a Creative Commons Attribution NonCommercial 3.0 License (CC BY-NC 3.0).

(C) Copyright A. Lax et al., 2015

Licensee PAGEPress, Italy

Italian Journal of Medicine 2015; 9:109-115

doi:10.4081/itjm.2015.395 in patients with stable chronic lung disease such as obstructive sleep apnea, the overlap syndrome [chronic obstructive pulmonary disease (COPD) and obstructive sleep apnea], neuromuscular disorders, obesityhypoventilation syndrome, and in other conditions such as sleep disorders associated with congestive heart failure (Cheyne-Stokes respiration). ${ }^{1}$ In this review we discuss the different types of NPPV, the specific conditions in which they can be used and the indications, recommendations and evidence supporting the efficacy of NPPV.

\section{Specific conditions for non-invasive ventilation}

\section{Obstructive sleep apnea-hypopnea syndrome}

The obstructive apnea-hypopnea syndrome (OSA) has an incidence of $2 \%$ in women and $4 \%$ in men. It is characterized by recurrent episodes of partial (hypopnea) or complete (apnea), obstruction of the upper airway during sleep, and it is associated with episodes of arousal and/or oxyhemoglobin desaturation. ${ }^{2}$

Symptoms of the syndrome are reported in Table 1.

The pathophysiology of obstructive sleep apnea is still controversial. Obesity, the classic hallmark in OSA, is not well understood as a cause leading to obstruction of the upper airways. Possible hypotheses include adipose tissue infarction of the tongue and/or the dilator muscles of the pharynx. The upper airway becomes less efficient, reducing oropharyngeal space especially at the end of exhalation. As a result, at the beginning of the next inspiration the dilator muscles 
of the pharynx (see genioglossus) should produce a greater contraction to overcome the tendency of the pharyngeal wall to collapse (due to the negative pressure inside the cavity and pharynx). The supine position is dangerous obstruction as the tongue tends and occludes the rear wall of the oropharynx.

This syndrome has been associated with the development of hypertension, coronary artery disease, cardiac arrhythmias, bleeding disorders, stroke and increased risk of sudden death during sleep. It is also associated with a higher rate and greater severity of traffic accidents, increased use of health care facilities and reduced capacity for work. ${ }^{3}$ Strong evidence exists that non-invasive ventilation, most often, continuous airway positive pressure (CPAP) has significant advantages in this type of disease, improving sleep quality, daytime wakefulness, and cognitive function. Consequently, the quality of life improves. These improvements are wide-ranging: reduction of traffic accidents, lower arterial blood pressure and reduction in the morbidity and mortality rates of myocardial infarction and stroke demonstrate the wide spectrum of CPAP's benefits. ${ }^{4,5}$

\section{Complex sleep apnea}

Complex sleep apnea syndrome is a type of central apnea characterized by the development or persistence of central apneas or hypopneas during the application of CPAP. These patients manifest predominantly obstructive apneas during diagnostic sleep studies. Probably the cause of the syndrome is the application of high CPAP pressure in obstructive sleep apnea patients with enhanced chemoreceptor sensitivity or in patients with sleep fragmentation. ${ }^{6}$ Treatment modalities consist in the avoidance of high pressure and permissive flow limitation, and advanced ventilation techniques: bilevel-positive airway pressure ventilation in the spontaneous-timed mode or adaptive servo-ventilation (ASV). ${ }^{6}$

\section{Sleep-disturbances associated with cardiac dysfunction}

The prevalence of obstructive sleep apnea in patients with impaired left ventricular ejection fraction is estimated about $11 \%$ to $53 \%$. It is also known that the sleep obstructive apnea-hypopnea syndrome can worsen a state of congestive heart failure, by causing a periodic increase in negative intrathoracic pressure, by raising arterial blood pressure, and causing tachycardia from sympathetic nervous system stimulation from hypoxia, hypercapnia and arousals. ${ }^{7}$

CPAP treatment produces a reduction in blood pressure and improves left ventricular systolic function in patients with chronic heart failure and obstructive sleep apnea. Recent studies in patients with chronic heart failure associated with obstructive sleep apnea have shown a further improvement of cardiac function in patients treated with bilevel positive airway pressure ventilation (BIPAP). ${ }^{8}$

The periodic breathing, Cheyne-Stokes respiration is a particular variety of central sleep apnea which is frequently associated with congestive heart failure.

Central sleep apnea (CSA) is associated with periodic breathing. Cheyne-Stokes breathing in heart failure is often the initial factor that, through mechanisms such as high chemo-sensitivity and prolonged circulation time, determines the onset of apnea. CSA with its characteristic desaturation apnea-relate and sympathetic hyperactivity tends to worsen the prognosis of heart failure. CSA is characterized by cessation of respiratory drive during sleep, cessation of airflow obstruction and impaired gas exchange. Unlike the OSA in which there is a respiratory effort to overcome the resistance of the upper airway, CSA is characterized by the absence of respiratory movement due to the cessation of ventilation. In the heart failure patients, the onset of apnea occurs by a redistribution of blood volume from the lower limbs to pulmonary circulation that is mainly triggered by the supine position. Stimulation of pulmonary vagal receptors causes hyperventilation which results in hypocapnia.

When the value decreases below the hypocapnic apneic threshold, stimulation of the bulbar center ceases, inspiratory drive stops, and apnea occurs. In patients with chronic heart failure, the prolonged circulation time due to the reduction in cardiac output leads to a delay of feedback between chemoreceptors and bulbar centers resulting in hyperventilation and respiratory greater instability. The main risk factors for CSA are male sex, hypocapnia, atrial fibrillation and advanced age. CPAP and BIPAP can fail to correct this category of apneas; therefore, a servo-assisted mode (ASV or adaptive servo-ventilation) is recommended. ${ }^{9}$

\section{Obesity-hypoventilation syndrome}

Obesity hypoventilation refers to a syndrome including daytime hypercapnia $\left(\mathrm{PCO}_{2}>45 \mathrm{mmHg}\right)$ in

Table 1. Typical symptoms of obstructive apnea-hypopnea syndrome.

\section{Snore}

Nocturia

Unrefreshing sleep

Choking

Daytime sleepiness

Decreased libido

Morning headache

Enuresis 
obese people in which no other cause of hypoventilation is present. Its prevalence among patients with obstructive sleep apnea is $20 \%$ to $30 \%$ and is greater in extremely obese patients (body mass index $>40$ ). Approximately $10 \%$ of patients with obesity-hypoventilation syndrome do not have sleep apnea-hypopnea syndrome. Additionally, nocturnal hypoxemia and diurnal hypercapnia persist in about $40 \%$ of these patients after the treatment with CPAP eliminated apnea. Factors other than sleep apnea contribute to the development of obesity-hypoventilation syndrome associated with the persistence of daytime hypercapnia: these include body mass index and apnea-hypopnea index, mean overnight oxygen saturation and the severity of restrictive ventilatory syndrome. BIPAP therapy may be useful in those patients in whom CPAP has failed or gave unsatisfactory results. The average volume-assured pressure support ventilation seems to be able to lower $\mathrm{PCO}_{2}$ more compared to BIPAP, but it is not able to further improve the oxygenation, sleep quality or quality of life. ${ }^{10}$

\section{Neuromuscular and chest wall disorders}

Non-invasive ventilation has been used in patients with progressive neuromuscular disease or serious abnormalities of the thoracic cage, with recognized benefits, including an improved survival rate and an improved quality of life. The benefits of non-invasive ventilation in this type of patient includes improvements of the daytime levels of blood gas (including hypercapnia), a reduction in the oxygen cost of breathing, an increase in the ventilatory response to increased carbon dioxide, and improved lung compliance. ${ }^{11}$

\section{Chronic obstructive pulmonary disease and sleep apnea (overlap syndrome)}

Sleep-disordered breathing (mainly obstructive sleep apnea) and COPD are the most common lung diseases: a large number of patients have both disorders, hence the term overlap syndrome. The overlap syndrome was first described by Flenley in 1985 as a combination of COPD and obstructive apnea-hypopnea syndrome. The coexistence of these conditions can lead to severe episodes of desaturation during sleep, thus increasing the risk of hypoxemia, daytime hypercapnia and pulmonary hypertension. Non-invasive ventilation may be useful in patients with overlap syndrome, but there are no controlled studies. ${ }^{12,13}$

\section{Clinical criteria for initiating non-invasive ventilation}

The presence of symptoms and physiological markers of hypoventilation are useful in identifying the clinical severity; moreover, these factors relate to therapeutic decision-making, especially initiating nocturnal non-invasive ventilation). ${ }^{1}$ In a typical progressive disease two successive steps occur: i) initial phase of nocturnal hypoventilation reversible during waking hours, associated with few or no clinical symptoms; ii) nocturnal and daylight hypoventilation associated with clinical symptoms which shows a reduced respiratory reserve.

The continuous sleep monitoring of $\mathrm{PCO}_{2}$ and $\mathrm{O}_{2}$ saturation values are necessary to document the presence of nocturnal hypoventilation which may be present in all the stages of sleep (in some cases only during REM sleep). Daytime hypoventilation is defined by reduced values of arterial oxygen tension $\left(\mathrm{PaO}_{2}\right)$, high levels of arterial carbon dioxide tension $\left(\mathrm{PaCO}_{2}\right)$ and/or high serum bicarbonate levels with a relatively normal $\mathrm{pH}$. Chronic daytime hypoventilation is an important indicator always associated with nocturnal hypoventilation. In the presence of daytime hypoventilation, polysomnography is carried out to exclude obstructive or central apnea. Clinical symptoms, although modest, should be evaluated carefully, because they are very important in determining disease severity and prognosis as well defining the need for non-invasive ventilation. Pulmonary function tests may be helpful in defining the reduction of lung function, but they have a low predictive value for patients with sleep-related hypoventilation. However, in patients with neuromuscular disease, there is a good correlation between lung function and nocturnal hypoventilation: it has been shown that hypoventilation during REM only or during all sleep stages or in the daytime, appears respectively with supine inspiratory vital capacities of less than $40 \%$, $25 \%$ or $12 \%$ of predicted values. ${ }^{1,14}$

\section{Types of non-invasive ventilation}

\section{Continuous positive airway pressure}

CPAP is currently the most widely used mode of non-invasive ventilation in the treatment of obstructive sleep-disordered breathing and of disordered breathing associated with chronic heart failure. It consists in the application of a constant level of positive pressure during spontaneous breathing. The mechanism of action of CPAP includes a series of actions on pathophysiological mechanisms: i) it prevents intermittent narrowing and collapse of the airways in patients with obstructive sleep apnea-hypopnea syndrome; ii) it counteracts auto-positive end-expiratory pressure, which reduces respiratory muscles load, the work of breathing and daytime $\mathrm{PaCO}_{2}$ in patients with overlap syndrome; iii) it improves lung function, particularly the functional residual capacity, daytime gas exchange in patients with obstructive sleep apnea-hypopnea syndrome; iv) it improves systolic function of the left ventricle in patients with heart fail- 
ure coexisting with obstructive sleep apnea-hypopnea syndrome. ${ }^{4}$

\section{Automatic adjustment of the level of continuous positive airway pressure}

Automatic adjustment of the level of CPAP (autoCPAP) is delivered via a self-titrating CPAP device, which uses algorithms to detect variations in the degree of obstruction and adjusts the pressure level to restore normal breathing. Auto-CPAP compensates for factors that modify the upper airway collapsibility, such as body posture during sleep, stage of sleep, use of alcohol, and drugs that affect upper airway muscle tone. ${ }^{4}$ It is not recommended in the diagnosis of OSA and titrating continuous pressure during split-night. The auto-CPAP can be used during polysomnography or cardiorespiratory monitoring to titrate a single pressure value to be used later with fixed CPAP for treatment of OSA in patients without comorbid conditions. The use of auto-CPAP is reserved only for those patients with sleep apnea syndrome only present during REM or respiratory events related to position, in whom constraining positional maneuvers are poorly tolerated. ${ }^{15}$

\section{Servo-assisted ventilation (or adaptive servo-ventilation)}

The servo-assisted ventilation has been developed for the treatment of Cheyne-Stokes respiration-central apnea syndrome in patients with chronic heart failure who have a breathing pattern characterized by periods of crescendo-decrescendo change in tidal volume with possible intercalated episodes of central apnea or hypopnea. This more complex device can use patient expiratory positive airway pressure (EPAP) level sufficient to control the obstructive apnea. The device then automatically adjusts the inspiratory pressure support for each inspiration within a pre-specified range, to maintain a moving-target ventilation set at $90 \%$ of the patient's recent average ventilation. The aim is the stabilization of breathing patterns and to reduce the respiratory alkalosis that can trigger apnea reentry cycles. ${ }^{16}$

\section{Bilevel positive airway pressure}

Bilevel positive airway pressure is also used for sleep-related disorders (including those associated with chronic heart failure), but its main indication is in pathological conditions associated with hypoventilation. The BIPAP devices deliver a higher pressure during inspiration [inspiratory positive airway pressure (IPAP)] and a lower pressure during expiration (EPAP). The gradient between IPAP and EPAP (pressure support ventilation) is crucial in maintaining adequate alveolar ventilation and reducing $\mathrm{PaCO}_{2}$. The IPAP acts also in reducing the work of breathing and fatigue, reducing the workload of respiratory muscles; EPAP has the function of maintaining the patency of the upper airway, to control obstructive apnea and to improve the functional residual capacity. BIPAP is now proposed for the type of patients who require high expiratory pressures to control obstructive sleep apnea-hypopnea, but who cannot tolerate exhaling against a high-fixed CPAP pressure. ${ }^{17}$ Other indications of BIPAP are the treatment of coexisting central apnea or hypoventilation, the obesity-hypoventilation syndrome, that cannot have a complete correction of the hypoxic state with CPAP only, the overlap syndrome and neuromuscular disorders. Although the patient should be able to maintain spontaneous breathing, it is used to set a back-up rate option for those patients whose ventilation during sleep may be particularly impaired (neuromuscular disorders, complex sleep apnea, central apnea in chronic heart failure, obesity-hypoventilation syndrome). ${ }^{4,17}$

\section{Average volume-assured pressure support ventilation}

Average volume-assured pressure support ventilation is used in patients with chronic hypoventilation and in particular with obesity hypoventilation syndrome, neuromuscular diseases, and sometimes, in chronic obstructive pulmonary disease. In this mode a target tidal volume is set; the device adjusts the pressure support to reach the set tidal volume. The advantage of this mode (a cross between the volume and pressure-assisted mode) is that it guarantees a delivered tidal volume adjusted despite variability in the patient effort, airway resistance, and lung or chest wall compliance. A particular benefit of this mode is that it may be modified as the disease progresses (as it occurs in neuromuscular disorders such as amyotrophic lateral sclerosis). ${ }^{4}$ All the types of ventilation are summarized in the Table 2.

\section{Management of non-invasive mechanical ventilation}

\section{Initiation and settings in case of nocturnal ventilation}

The main objective of the use of non-invasive ventilation is the correction of blood gas values near normal with the least possible discomfort or sleep disturbance. Even if there is no absolute recommendation, it is good practice to proceed in three successive steps. The first step is to choose and adjust the ventilator settings while the patient is awake, assuring physiological adequacy and patient comfort for at least one or two hours. In the second step the clinician should evaluate the adequacy of the settings when sleeping during a nap and a night's sleep. Different op- 
tions, according to the resources available in each center, are used. A full polysomnography recording oxygen saturation $\left(\mathrm{SpO}_{2}\right)$ and transcutaneous $\mathrm{PCO}_{2}$ $\left(\mathrm{PtcCO}_{2}\right)$ or end-tidal $\left(\mathrm{PetCO}_{2}\right)$, flow, tidal volume, airway pressure, rib cage and abdomen excursion as well as sleep-staging allows a complete assessment (gold standard). When the resources are not available fewer parameters may be used. Nocturnal oximetry or simplified sleep monitoring (oro-nasal airflow, heart rate, oxygen saturation, body position) allow indirect identification of respiratory events concerning the sleep-related breathing disorders through oximetry (desaturation events) but these measurements are not able to distinguish between obstructive and central events, because of the absence of registration of movements of the chest and abdomen. This limit does not permit a correct setting of a ventilatory therapy.

The second step relates to patient tolerance, comfort, changes in sleep quality and well-being; these data should be obtained. The third step consists of looking for reduction in $\mathrm{PaCO}_{2}$ and augmentation of $\mathrm{PaO}_{2}$ without dyspnea during the day in free ventilation after sev-

Table 2. Types of non-invasive positive pressure ventilation.

Continuous positive airway pressure

Applications: obstructive sleep apnea; congestive heart failure with coexisting obstructive sleep apnea; obesity-hypoventilation syndrome with coexisting obstructive sleep apnea

Setup requirements: CPAP level

Advantages: simple to use; relatively inexpensive

Disadvantages: minimal or no ventilation support; preset pressures may not address variability in obstructive sleep apnea, severity with sleep stages and positional stages

Auto-CPAP

Applications: obstructive sleep apnea; congestive heart failure with coexisting obstructive sleep apnea; obesity-hypoventilation syndrome with coexisting obstructive sleep apnea

Setup requirements range of allowable CPAP levels

Advantages: reduces the number of titration studies; self-adjusting to adapt to variability in obstructive sleep apnea with sleep stages and positional changes; maybe useful for patients with ongoing weight loss such as after bariatric surgery

Disadvantages: more expensive than fixed CPAP; may not be effective for patients with cardiopulmonary disorders or other conditions in which desaturation may be unrelated to obstructive events

Adaptive servo-ventilation

Applications: congestive heart failure; central sleep apnea; complex sleep apnea syndrome

Setup requirements: maximum and minimum inspiratory pressures; end-expiratory pressure

Advantages: adapts pressure to maintain more consistency of respiration over time

Disadvantages: more expensive than other modes; may worsen ventilation in disease with chronic ventilator insufficiency such as COPD or restrictive thoracic disorders

Bilevel positive airway pressure

Without backup rate

Applications: obstructive sleep apnea with CPAP intolerance; obstructive sleep apnea with central sleep apnea; restrictive thoracic disorders; severe chronic obstructive pulmonary disease; obesity hypoventilation syndrome with coexisting obstructive sleep apnea and residual hypoventilation despite CPAP

Setup requirements: inspiratory and expiratory positive airway pressures

Advantages: promotes alveolar ventilation; unloads respiratory muscles; decreases the work of breathing; controls obstructive hypopneas

Disadvantages: more expensive than CPAP; may generate central apnea

With backup rate

Applications: central sleep apnea; complex sleep apnea syndrome; worsening restrictive disorder

Setup requirements: inspiratory and expiratory positive airway pressure; backup rate; ratio of inspiratory time to expiratory time

Advantages: provides mandatory respiratory support during central or pseudo-central apneas

Disadvantages: more expensive than conventional BIPAP; may generate central apnea

Average volume-assured pressure support

Applications: obesity-hypoventilation syndrome; neuromuscular disease; chronic obstructive pulmonary disease

Setup requirements: target tidal volume $(8 \mathrm{~mL} / \mathrm{kg}$ of ideal weight); inspiratory positive airway pressure limits; respiratory rate

Advantages: ensures a delivered tidal volume; compensates for diseases progression

Disadvantages: more expensive than other modes

CPAP, continuous positive airway pressure; BIPAP, bilevel positive airway pressure. 
eral nights of NPPV. This is done to confirm that the settings are adequate for the patient's needs. ${ }^{1,18}$

If the results are not satisfactory, changes must be made to the settings. One may also change the type of mask and ventilator. At the beginning a starting level of pressure support of $10 \mathrm{~cm} \mathrm{H}_{2} \mathrm{O}$ is recommended. Continuing the adaptation, the pressure level can progressively be increased to achieve evidence of improvement. Pressure support higher than $20 \mathrm{~cm} \mathrm{H}_{2} \mathrm{O}$ is rarely necessary. ${ }^{1,18,19} \mathrm{~A}$ back-up frequency set close to the spontaneous frequency of the patient during sleep is a reasonable substitute to inspiratory trigger failure to avoid central apnea induced by transitory but repeated hyperventilation passing the apnea threshold. When employing a volume-preset ventilator, the initial suggested setting may be established by adjusting the frequency of ventilator-delivered breaths so that it approximates the patient's spontaneous breathing frequency during sleep, an inspiratory time/total breathing time between 0.33 and 0.5 and a relatively high tidal volume of around $10-15 \mathrm{~mL} / \mathrm{kg}$ to insure sufficient tidal volume in case of leaks. ${ }^{1}$

Supplemental oxygen $\left(\mathrm{O}_{2}\right)$ will be added to the ventilator circuit, immediately near the interface or hooked to a respirator, especially in those patients who require oxygen during the daytime (COPD, cystic fibrosis, bronchiectasis). In the absence of obstructive pulmonary disease, the addition of $\mathrm{O}_{2}$ to the ventilation circuit may be justified only to maintain an acceptable level of $\mathrm{PaO}_{2}$ during sleep and only after all the parameters have been optimized. ${ }^{20}$

\section{Follow-up}

Clinical follow-up and daytime arterial blood gases should be conducted at least twice a year. The recordings during sleep (possibly identical to those performed for the adaptation to non-invasive ventilation), are useful. At any time, when there are indications of unsatisfactory results such as the recurrence of clinical symptoms and/or signs of hypoventilation on arterial blood gases, inadequate non-invasive ventilation should be suspected, and a complete objective assessment of ventilation during sleep with polysomnography must be undertaken.

When the non-invasive ventilation is not proven to be optimal, a change of ventilation modality and/or parameters of the ventilator and/or a revision of the interface may be indicated. In case of disease progression one should be considered increasing the duration of ventilation during the day. The interfaces need to be regularly checked and modified or adapted to the changing needs of the patient. ${ }^{1}$

\section{Management of complications and side effects}

\section{Air leaks during ventilation}

The major potential adverse effect is the loss of effectiveness of the ventilation and therefore the potential fragmentation of sleep. A variety of more or less effective measures have been suggested to tackle the problem of leaks during NPPV. These include the prevention of neck flexion, the semi-recumbent positioning of the patient, the use of a chin rest or a cervical collar to prevent opening of the mouth, switching to controlled pressure mode, decreasing the peak inspiratory pressure and increasing the delivered volume, optimizing the interface (using oro-nasal masks if possible). The effectiveness of each of these measures must be confirmed during sleep recording. ${ }^{21}$

\section{Nasal dryness, congestion}

As shown in the CPAP literature, the side effects of nasal dryness, congestion, and rhinitis are related to a defect of humidification. For the patients with nasal and mouth dryness, a cold pass over or a heated humidifier can be used. ${ }^{22,23}$

\section{Aerophagia}

Aerophagia (swallowing air) is frequently reported but is rarely intolerable. Minor clinical signs are eructation, flatulence and abdominal discomfort. Aerophagia usually depends on the level of inspiratory pressure and decreases if the peak inspiratory pressure is maintained below $25 \mathrm{~cm} \mathrm{H}_{2} \mathrm{O}$ pressure ${ }^{23}$ (Table 3).

Table 3. Management of complications and side effects of non-invasive ventilation.

\begin{tabular}{|c|c|}
\hline Complication and/or side effect & Action \\
\hline Air leaks & $\begin{array}{l}\text { Prevention of neck flexion } \\
\text { Semi-recumbent positioning } \\
\text { Use of chin rest } \\
\text { Use of cervical collar } \\
\text { Switch to controlled pressure mode } \\
\text { Decrease peak inspiratory pressure and increase volume } \\
\text { Optimize the interfaces (using oro-nasal mask) }\end{array}$ \\
\hline Nasal dryness, congestion & $\begin{array}{l}\text { Cold pass over } \\
\text { Heated humidifier }\end{array}$ \\
\hline
\end{tabular}

Aerophagia, eructation, flatulence, abdominal discomfort

Decrease peak inspiratory pressure below $25 \mathrm{cmH}_{2} \mathrm{O}$ 


\section{Conclusions}

In conclusion:

- Optimizing patient acceptance and adherence to non-invasive ventilation treatment is challenging. The treatment of sleep-related disorders is a lifethreatening condition. The optimal level of treatment should be determined in a sleep laboratory. ${ }^{1}$

- Side effects directly affecting the patient's adherence to treatment are known. The most common are nasopharyngeal symptoms including increased congestion and rhinorrhea; these effects are related to reduced humidity of inspired gas. Humidification of delivered gas may improve these symptoms. ${ }^{24}$ Sleep specialists should review the results of objective testing with the patient. Education of the patient concerning the nature of the disorder and treatment options is mandatory. ${ }^{24}$

- The impact of weight loss, sleep position, alcohol avoidance, risk factor modification and medication effects should be discussed..$^{10}$ The patient should be counseled on the risks and management of drowsy driving. Patient education should optimally be delivered as a part of a multidisciplinary chronic disease management team. ${ }^{25}$

\section{References}

1. Robert D, Argaud L. Non-invasive positive ventilation in the treatment of sleep-related breathing disorders. Sleep Med 2007;8:441-52.

2. Cassel W, Canisius S, Becker HF, et al. A Prospective polysomnographic study on the evolution of complex sleep apnoea. Eur Respir J 2011;38:329-37.

3. Parati G, Lombardi C, Hedner J, et al. Recommendations for the management of patients with obstructive sleep apnoea and hypertension. Eur Resp J 2013;41:523-38.

4. Teerhakittikul T, Ricaurte B, Aboussouan LS. Non-invasive positive pressure ventilation for stable outpatients: CPAP and beyond. Cleve Clin J Med 2010;77: 705-14.

5. Marin JM, Carrizo SJ, Vicente E, Agusti AG. Long-term cardiovascular outcomes in men with obstructive sleep apnoea-hypopnea with or without treatment with continuous positive airway pressure: an observational study. Lancet 2005;365:1046-53.

6. Verbraecken J. Complex sleep apnea syndrome. Breathe 2013;9:373-80.

7. Bordier P. Sleep apnoea in patients with heart failure. Part I: diagnosis, definitions, prevalence, pathophysiology and haemodynamic consequences. Arch Cardiovasc Dis 2009;102:651-61.

8. Mansfield DR, Gollogly NC, Kaye DM, et al. Controlled trial of continuous positive airway pressure in ob- structive sleep apnea and heart failure. Am J Resp Crit Care Med 2004;169:361-6.

9. Tkacova R, Hall MJ, Liu PP, et al. Left ventricular volume in patients with heart failure and Cheyne-Stokes respiration during sleep. Am J Resp Crit Care Med 1997; 156:1549-55.

10. Mokhlesi B. Obesity hypoventilation syndrome: a stateof-the-art review. Resp Care 2010;55:1347-65.

11. Nickol AH, Hart N, Hopkinson NS, et al. Mechanisms of improvement of respiratory failure in patients with restrictive thoracic disease treated with non-invasive ventilation. Thorax 2005;60:754-60.

12. Hill NS. Non-invasive ventilation for chronic obstructive pulmonary disease. Resp Care 2004;49:72-87.

13. McEvoy RD, Pierce RJ, Hillman D. Australian trial of non-invasive ventilation in chronic airflow limitation (AVCAL) Study group. Nocturnal non-invasive nasal ventilation in stable hypercapnic COPD: a randomized controlled trial. Thorax 2009;64:561-6.

14. Lo Coco D, Marchese S, Corrao S, et al. Development of chronic hypoventilation in amyotrophic lateral sclerosis patients. Resp Med 2006;100:1028-36.

15. Meurice JC, Cornette A, Philip-Joet F. Evaluation of auto CPAP devices in home treatment of sleep apneahypopnea syndrome. Sleep Med 2007;8:159-64.

16. Teschler H, Dohring J, Wang YM, Berthon-Jones M. Adaptive pressure support servo-ventilation: a novel treatment for Cheyne-stokes respiration in heart failure. Am J Resp Crit Care Med 2001;164:614-9.

17. Kushida CA, Littner MR, Hirshkowitz M. American Academy of Sleep Medicine. Practice parameters for the use of continuous and bilevel positive airway pressure devices to treat adult patients with sleep related patients breathing disorders. Sleep 2006;29:375-80.

18. Epstein LJ, Kristo D, Strollo PJ Jr, et al. Clinical guideline for the evaluation, management and long-term care of obstructive sleep apnea in adults. J Clin Sleep Disord 2009;5:263-76.

19. Rosario IC. Obstructive sleep apnea: a review and update. Minn Med 2011;94:44-8.

20. Schwartz AR, Kacmarek RM, Hess DR. Factors affecting oxygen delivery with bi-level positive airway pressure. Resp Care 2004;49:270-5.

21. Teschler H, Stampa J, Ragette R, et al. Effect of mouth leak on effectiveness of nasal bilevel ventilator assistance and sleep architecture. Eur Resp J 1999;14:1251-7.

22. Randerath WJ, Meier J, Genger H, et al. Efficiency of cold Passover and heated humidification under continuous positive airway pressure. Eur Resp J 2002;20:183-6.

23. Hill NS. Complications of noninvasive ventilation. Respir Care 2000;45:480-1.

24. Qaseem A, Holty JE, Owens DK, et al. Management of obstructive sleep apnea in adults: a clinical practice guideline from American College of Physician. Ann Intern Med 2013 [Epub ahead of print].

25. Soares Pires F, Drummond M, Marinho A, et al. Effectiveness of a group education session on adherence with APAP in obstructive sleep apnea-a randomized controlled study. Sleep Breath 2013;17:993-1001. 